\title{
Estratégias de cuidado às gestantes e puérperas usuárias de substâncias psicoativas: revisão integrativa
}

\author{
Care strategies for pregnant and puerperal psychoactive substances users: an integrative review
}

Estrategias de atención para mujeres embarazadas y puérperas usuarias de sustancias psicoactivas: revisión integradora

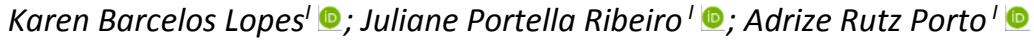

'Universidade Federal de Pelotas, Pelotas, RS, Brasil

\begin{abstract}
RESUMO
Objetivo: analisar a produção de conhecimento acerca das estratégias de cuidado direcionadas às gestantes e puérperas usuárias de substâncias psicoativas. Método: revisão integrativa da literatura realizada em julho de 2019, nas bases de dados PubMed, BDEnf, LILACS e SciELO, considerando os critérios de inclusão: artigos científicos disponíveis online na íntegra; com resumo disponível; nos idiomas português, inglês e espanhol. Resultados: foram levantados 16 artigos, dos quais cinco compuseram a revisão. Como estratégias de cuidado, identificaram-se: captação precoce na atenção pré-natal, ações de educação em saúde, acompanhamento nutricional, Terapia de Substituição e Terapia Cognitivo Comportamental. Conclusão: os artigos analisados apontam a necessidade de educação permanente de profissionais de saúde, no sentido de qualificar a captação precoce e ofertar acolhimento sensível às demandas e especificidades de saúde de gestantes e puérperas usuárias de substâncias psicoativas.
\end{abstract}

Descritores: Cuidado Pré-Natal; Cuidado Pós-Natal; Usuários de Drogas; Terapêutica; Cuidado.

\section{ABSTRACT}

Objective: to examine knowledge production on care strategies for pregnant and puerperal psychoactive substance users. Method: in July 2019 this integrative literature review searched the PubMed, BDEnf, LILACS, and SciElo databases with the following inclusion criteria: complete scientific articles available online; with abstract accessible; in Portuguese, English, and Spanish. Results: 16 articles were identified, five of which entered the revision. Care strategies identified were: early identification in prenatal care, health education actions, nutritional monitoring, substitution therapy, and cognitive behavioral therapy. Conclusion: the articles examined pointed to the need for continuing professional development for health professionals with a view to improving early identification and offering sensitive receptiveness to the specific health demands and characteristics of pregnant and puerperal psychoactive substance users.

Descriptors: Prenatal Care; Postnatal Care; Drug Users; Therapeutics; Care.

\section{RESUMEN}

Objetivo: examinar la producción de conocimiento sobre estrategias de atención a las usuarias de sustancias psicoactivas embarazadas y puerperales. Método: en julio de 2019 esta revisión integradora de la literatura buscó en las bases de datos PubMed, BDEnf, LILACS y SciElo con los siguientes criterios de inclusión: artículos científicos completos disponibles en línea; con resumen accesible; en portugués, inglés y español. Resultados: se identificaron 16 artículos, cinco de los cuales ingresaron a la revisión. Las estrategias de atención identificadas fueron: identificación temprana en la atención prenatal, acciones de educación en salud, monitoreo nutricional, terapia de sustitución y terapia cognitivo-conductual. Conclusión: los artículos examinados señalaron la necesidad de un desarrollo profesional continuo de los profesionales de la salud con miras a mejorar la identificación precoz y ofrecer receptividad sensible a las demandas y características específicas de salud de las usuarias de sustancias psicoactivas embarazadas y puerperales.

Descriptores: Atención Prenatal; Atención Posnatal; Consumidores de Drogas; Terapéutica; Atención.

\section{INTRODUÇÃO}

O uso abusivo de sustâncias psicoativas esteve em significativa ascensão nas últimas décadas, tornando-se um problema complexo global de saúde pública, além de ser um desafio para governantes e principalmente para profissionais de saúde ${ }^{1}$. Trata-se de uma preocupação da Organização das Nações Unidas, elencada na agenda de 2030, pela necessidade de reforçar a prevenção e o tratamento do uso de substâncias, incluindo o abuso de drogas entorpecentes e o uso nocivo de álcool ${ }^{2}$.

Ressalta-se que pesquisadores apontam uma mudança no perfil dos consumidores de substância psicoativas, com diminuição da proporção entre homens e mulheres de um modo geral ${ }^{3,4}$. As estatísticas mostram que $85 \%$ das mulheres que usam substâncias psicoativas estão na idade fértil, utilizando inclusive no período gravídico-puerperal ${ }^{3}$.

Uma pesquisa realizada com 1.447 gestantes, cujo objetivo era de analisar a prevalência e fatores associados ao uso de drogas ilícitas na gestação, apontou que 1,45\% das mulheres faziam uso de substâncias psicoativas (canabinoides

Agradecimento à Fundação de Amparo à Pesquisa do Estado do Rio Grande do Sul (FAPERGS), Scientific Iniciation Scholar - Call CPESQ № 003/2019. Autora correspondente: Juliane Portella Ribeiro. E-mail: ju ribeiro1985@hotmail.com

Editora responsável: Cristiane Helena Gallasch 
e/ou cocaína e seus derivados), 22,32\% de bebidas alcoólicas e 4,22\% utilizavam cigarro ${ }^{5}$. Esses dados corroboram com outro estudo realizado em uma unidade de referência da Atenção Primária no Acre, com uma amostra de 30 gestantes usuárias de substâncias psicoativas, o qual identificou que $60 \%$ dessas mulheres faziam uso de álcool e $30 \%$ de tabaco ${ }^{6}$. Outro estudo, desta vez de coorte, acompanhou 674 mulheres entre 2008 e 2012 e mostrou que cerca de $17 \%$ declararam uso durante o período puerperal, sendo que 63,9\% usaram álcool, 58,3\% tabaco, 9,2\% cocaína/crack e 4,6\% usaram maconha7.

Estudiosos apontam para um número expressivo de mulheres que utilizam substâncias psicoativas no período gravídico puerperal. Entretanto, poucas são identificadas, constituindo uma lacuna na detecção precoce ${ }^{8}$. Uma pesquisa com 25 gestantes atendidas em unidades Básicas de Saúde em Maringá evidenciou que os profissionais de saúde, de forma geral, não abordavam questões relacionadas com o uso de drogas - lícitas ou ilícitas - durante o

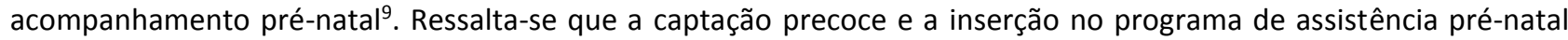
apresentam potencialidades no que tange a redução de complicações maternas e fetais ${ }^{10,11}$.

A utilização contínua e progressiva dessas substâncias interrompe o curso fisiológico do organismo, propiciando o surgimento de patologias como desnutrição e intercorrências obstétricas como abortamento, deslocamento prematuro da placenta, restrição de crescimento intrauterino, prematuridade, baixo peso ao nascer, síndrome de abstinência neonatal e problemas respiratórios fetais, culminando em elevados índices de mortalidade materna e neonatal ${ }^{11-13}$. No entanto, pesquisadores ressaltam que a maioria das gestantes usuárias de substâncias psicoativa não realiza acompanhamento, sendo captadas somente na hora do parto ou puerpério sem nenhum tipo de acompanhamento especializado ${ }^{14}$. Por essa razão, o uso de substâncias psicoativas por mulheres no período gravídico-puerperal tem sido considerado uma situação de emergência, mobilizando o Ministério da Saúde (MS) devido à gravidade de suas consequências tanto para a mulher como para o bebê.

Na Agenda Nacional de Prioridades de Pesquisa em Saúde, o MS aponta a necessidade de estudos no âmbito da saúde da mulher que considerem a prevalência e incidência de transtornos mentais especificamente na gravidez, parto e puerpério. Além disso, no que se refere a saúde mental dessa população, incentiva o desenvolvimento de pesquisas que abordem estratégias de prevenção e redução de danos para uso abusivo de substâncias psicoativas ${ }^{15}$.

Nesse sentido, considerando a lacuna existente no cuidado à mulher usuária de substâncias psicoativas no período gravídico-puerperal, o presente estudo teve como objetivo analisar a produção de conhecimento acerca das estratégias de cuidado direcionadas às gestantes e puérperas usuárias de substâncias psicoativas.

\section{MÉTodo}

Trata-se de revisão integrativa, que, pra sua execução, percorreram-se seis passos: escolha e definição do tema e da questão de pesquisa, estabelecimento de critérios de inclusão e exclusão de estudos, definição das informações a serem extraídas, análise crítica dos estudos incluídos no resultado, discussão dos resultados e apresentação da revisão integrativa ${ }^{16}$.

A revisão orientou-se a partir da seguinte questão: quais são as estratégias de cuidado direcionadas às gestantes e puérperas usuárias de substâncias psicoativas? Para conferir confiabilidade aos achados, efetivou-se dupla busca de artigos por diferentes pesquisadores, ambos doutores em enfermagem. Foram estabelecidos como critérios de inclusão: artigos científicos disponíveis online na íntegra; com resumo disponível, visando constatar a consonância do artigo com o objetivo do estudo; nos idiomas português, inglês e espanhol. Delimitou-se a busca por artigos a partir de 2011, pelo fato de que neste ano foi instituída a Rede Cegonha, que propõe o cuidado em rede, visando assegurar às mulheres a assistência humanizada na gravidez, no parto e no puerpério ${ }^{17}$. Como critério de exclusão: artigos não disponíveis gratuitamente.

A coleta de dados ocorreu no mês de julho de 2019 nas seguintes bases de dados: Public Medical (PubMed), Literatura Latino-Americana e do Caribe em Ciências da Saúde (LILACS), Bases de Dados da Enfermagem (BDEnf) e Scientific Eletronic Library Online (SciELO). Foram utilizando os Descritores em Ciências da Saúde (DeCS) e Medical Subject Headings (Mesh Terms): "Gravidez/Pregnancy/Embarazo", "Período Pós-Parto/Postpartum Period/Periodo Posparto", "Usuários de drogas/Drug Users/Consumidores de Drogas", "Terapêutica/Therapeutics/Terapéutica", "Promoção da Saúde/Health Promotion/Promoción de la Salud" e "Pré-Natal/Prenatal Care/Atención Prenatal", com os booleanos AND e OR de acordo com a estratégia apresentada (Figura 1):

Para a extração dos dados foi desenvolvido pelos pesquisadores um instrumento de coleta de dados, a fim de facilitar a caracterização dos artigos e realizar as análises. Tal instrumento continha as seguintes informações: identificação do artigo, bases de dados, ano de publicação, objetivos, delineamento do estudo, participantes, país de realização dos estudos, estratégias de cuidado e desfechos. 


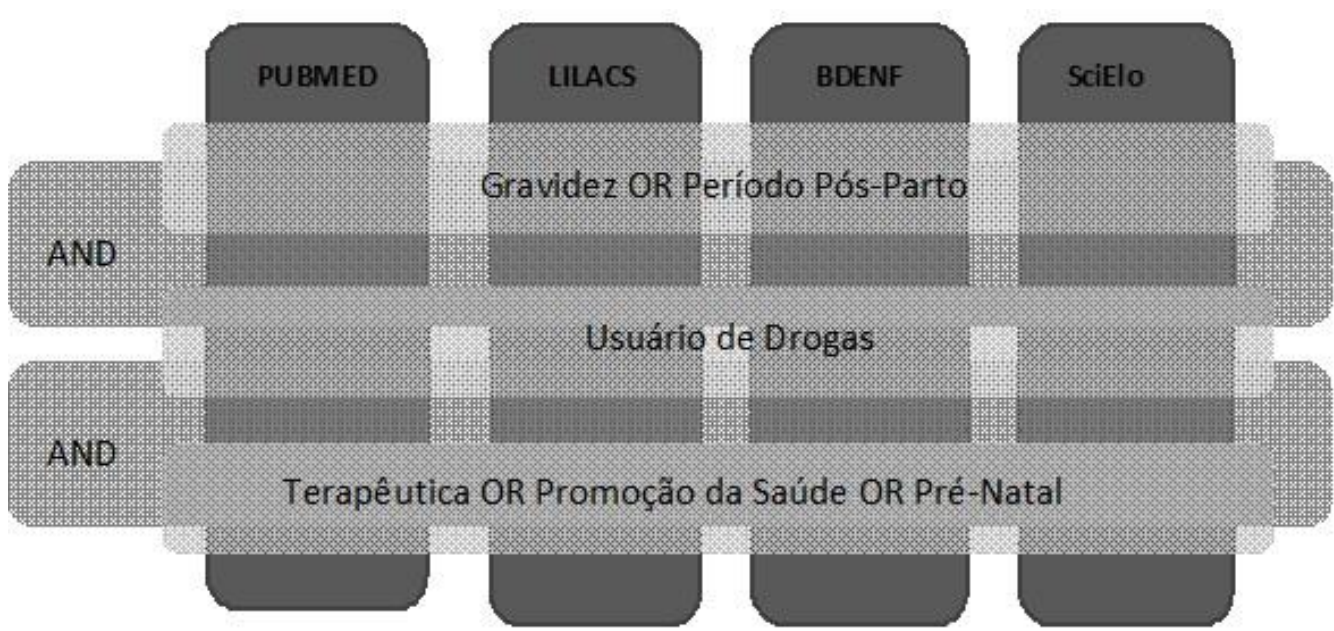

FIGURA 1. Estratégia de busca dos artigos científicos e estudos encontrados em cada base de dados. Pelotas, RS, Brasil, 2019.

\section{RESULTADOS}

Por meio de busca eletrônica foram levantadas 16 publicações nas quatro bases de dados utilizadas para essa revisão, sendo 15 artigos na PubMed e um artigo na LILACS; na BDEnf e na SciELO nenhum artigo foi localizado. A partir da leitura dos títulos, foram excluídas dez publicações que não responderam à questão de pesquisa; destaca-se que destes, um estava duplicado entre as bases de dados, reduzindo-se a seis artigos. Após a leitura dos resumos, foi excluída da revisão um artigo que não atendia aos critérios de inclusão, compondo assim a amostra do estudo em cinco artigos selecionados (Figura 2).

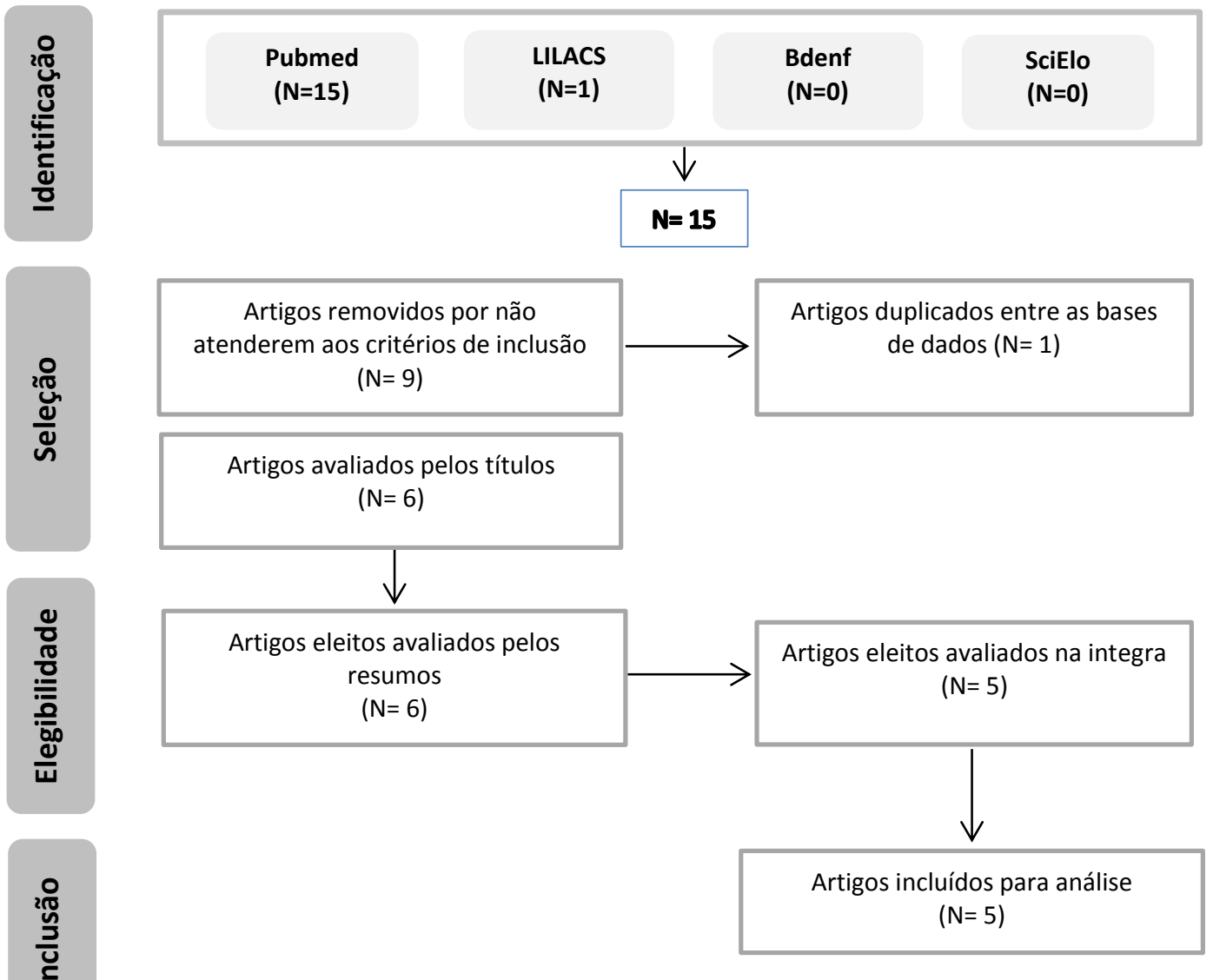

FIGURA 2: Fluxograma do processo de busca e seleção dos artigos. Pelotas, RS, Brasil, 2019. 
Entre os cinco artigos selecionados, três artigos foram publicados em periódicos multidisciplinares ${ }^{19-21}$, e os demais foram divulgados em periódicos de medicina ${ }^{18}$ e enfermagem ${ }^{9}$. Os artigos selecionados estão apresentados na Figura 3.

\begin{tabular}{|c|c|c|c|c|}
\hline $\begin{array}{c}\text { Titulo/ } \\
\text { Referencia* }\end{array}$ & $\begin{array}{l}\text { Ano/ } \\
\text { País }\end{array}$ & $\begin{array}{l}\text { Delineamento/ } \\
\text { Número de } \\
\text { participantes }\end{array}$ & $\begin{array}{l}\text { Estratégia de } \\
\text { Cuidado }\end{array}$ & Desfecho \\
\hline $\begin{array}{l}\text { Substance, structure } \\
\text { and stigma: Parents in } \\
\text { the UK accounting for } \\
\text { opioid substitution } \\
\text { therapy during the } \\
\text { antenatal and } \\
\text { postnatal periods }\end{array}$ & $\begin{array}{l}\text { 2013/ } \\
\text { Escócia }\end{array}$ & $\begin{array}{l}\text { Qualitativo } \\
19 \text { usuários } \\
\text { dependentes de } \\
\text { opióides }\end{array}$ & $\begin{array}{l}\text { Terapia de } \\
\text { Substituição }\end{array}$ & $\begin{array}{l}\text { A Terapia de Substituição de Opiáceos foi } \\
\text { associada neste estudo como uma forma de } \\
\text { proteção para a prole, passíveis com questões } \\
\text { sociais, como a custódia do filho, além de se } \\
\text { manter abstinentes, contribuindo para a redução } \\
\text { do estigma negativo de usuário de substância. }\end{array}$ \\
\hline $\begin{array}{l}\text { Costs of a } \\
\text { Motivational } \\
\text { Enhancement } \\
\text { Therapy Coupled } \\
\text { with Cognitive } \\
\text { Behavioral Therapy } \\
\text { versus Brief Advice } \\
\text { for Pregnant } \\
\text { Substance Users }\end{array}$ & $\begin{array}{l}\text { 2014/ } \\
\text { Estados } \\
\text { Unidos da } \\
\text { América }\end{array}$ & $\begin{array}{c}\text { Quantitativo } \\
168 \text { mulheres: } 82 \\
\text { receberam a } \\
\text { terapia de } \\
\text { aumento } \\
\text { motivacional e } 86 \\
\text { receberam o } \\
\text { aconselhamento } \\
\text { breve }\end{array}$ & $\begin{array}{c}\text { Terapia } \\
\text { Cognitiva } \\
\text { Comportamental }\end{array}$ & $\begin{array}{l}\text { A implementação de um programa de Terapia } \\
\text { Cognitiva Comportamental e aprimoramento de } \\
\text { terapia comportamental para promover a } \\
\text { abstinência de drogas em mulheres grávidas está } \\
\text { associada a custos modestos. Além disso, o uso } \\
\text { da técnica permite uma compreensão mais } \\
\text { abrangente da intervenção as mulheres que } \\
\text { abusam de substâncias. }\end{array}$ \\
\hline $\begin{array}{l}\text { Percepções e práticas } \\
\text { de gestantes atendidas } \\
\text { na atenção primária } \\
\text { frente ao uso de } \\
\text { drogas }^{9}\end{array}$ & $\begin{array}{l}\text { 2014/ } \\
\text { Brasil }\end{array}$ & $\begin{array}{l}\text { Qualitativo } \\
25 \text { mulheres } \\
\text { usuárias de } \\
\text { substâncias }\end{array}$ & $\begin{array}{c}\text { Ações de } \\
\text { educação em } \\
\text { saúde }\end{array}$ & $\begin{array}{l}\text { As gestantes apontaram que há fragilidade na } \\
\text { sensibilização da equipe para esclarecer a } \\
\text { importância da interrupção do uso de substâncias } \\
\text { na gestação; fragilidade na capacitação para } \\
\text { abordagem adequada e detecção precoce, que } \\
\text { vise a minimização de danos maternos-fetais. }\end{array}$ \\
\hline $\begin{array}{l}\text { A Qualitative Study of } \\
\text { Substance use during } \\
\text { Pregnancy: } \\
\text { Implications for } \\
\text { Reproductive } \\
\text { Healthcare in Western } \\
\text { Kenya }^{20}\end{array}$ & $\begin{array}{l}\text { 2016/ } \\
\text { Quênia }\end{array}$ & Qualitativo & $\begin{array}{c}\text { Captação precoce } \\
\text { na atenção pré- } \\
\text { natal }\end{array}$ & $\begin{array}{l}\text { O estudo aponta a importância de educação } \\
\text { cultural apropriada para os profissionais de } \\
\text { saúde, para a assistência qualificada e com } \\
\text { equidade. Também a necessidade de capacitação } \\
\text { clínica para utilização de questionários } \\
\text { estruturados como o Alcohol, Smoking and } \\
\text { Substance Involvement Screening Test (ASSIST), } \\
\text { Alcohol Use Disorders Identification Test (AUDIT) } \\
\text { e The Drug Abuse Screening Test (DAST); visando } \\
\text { busca ativa oportuna. Além de abordagem do } \\
\text { contexto social como gênero, violência e } \\
\text { vulnerabilidade social, compreendendo essa } \\
\text { mulher de forma integral, atendendo a outras } \\
\text { necessidades em saúde. }\end{array}$ \\
\hline $\begin{array}{l}\text { Dietary Intake Among } \\
\text { Opioid Dependent and } \\
\text { Alcohol Using Pregnant } \\
\text { Women }^{21}\end{array}$ & $\begin{array}{l}\text { 2018/ } \\
\text { México }\end{array}$ & $\begin{array}{l}\text { Quantitativo } \\
102 \text { gestantes }\end{array}$ & $\begin{array}{c}\text { Acompanhament } \\
\text { o nutricional }\end{array}$ & $\begin{array}{l}\text { O estudo demonstrou que mulheres grávidas } \\
\text { dependentes de opióides podem ter ingestão } \\
\text { inadequada de micronutrientes durante a } \\
\text { gravidez, no qual os programas de Tratamento } \\
\text { Medicamentoso Assistido devem considerar a } \\
\text { integração da avaliação nutricional como uma } \\
\text { possível intervenção, atentando para um } \\
\text { crescimento e desenvolvimento saudável para o } \\
\text { feto, além de um aporte adequado para essa } \\
\text { mulher. }\end{array}$ \\
\hline
\end{tabular}

FIGURA 3: Síntese dos artigos analisados que compõem o estudo - Pelotas, RS, Brasil, 2019.

Em relação ao ano de publicação, verificou-se que há uma lacuna de estudos que abordem as estratégias de cuidado direcionadas às gestantes e puérperas usuárias de substâncias psicoativas. No ano de 2013 houve apenas um artigo publicado, em 2014 apenas duas publicações, e entre 2016 e 2018 novamente apenas uma publicação por ano. Com relação à abordagem dos estudos, três eram qualitativos e dois quantitativos. Os participantes dos estudos apresentaram diversidade; de forma geral predominaram estudos com mulheres, seguidos de estudos com gestantes. Os locais de desenvolvimento das pesquisas foram: Brasil, México, Quênia, Escócia e Estados Unidos da América. 
A coleta de dados referida nos artigos apontou o uso de instrumentos como entrevistas, questionários, prontuários, sessões terapêuticas, coleta de amostra biológica e aplicação de testes padronizados como o Alcohol Use Disorder Identification Test-C (AUDIT-C). A análise dos respectivos estudos foi consoante aos métodos adotados, utilizando análises estatísticas como a Análise de Variância (ANOVA), o Teste de Fischer, o teste de sensibilidade individual e bidirecional, as análises descritivas, o Sistema Ecológico Social e a análise comparativa. Para as análises de alguns dados, foram empregados programas como o Software for Statistics and Data Science (STATA) e NVIVO, em variadas versões.

As estratégias de cuidado direcionadas às gestantes e puérperas usuárias de substâncias psicoativas apontadas nos estudos foram: captação precoce na atenção pré-natal, com acolhimento sensível às demandas e especificidades das usuárias; ações de educação em saúde; acompanhamento nutricional; Terapia de Substituição e Terapia Cognitivo Comportamental.

\section{DISCUSSÃo}

Os artigos analisados indicam certas estratégias de cuidado às gestantes e puérperas usuárias de substâncias psicoativas. Dentre elas, há a captação precoce na atenção pré-natal com acolhimento sensível às demandas e especificidades das usuárias, visto que uma das barreiras à adesão ao pré-natal é o despreparo de profissionais que realizam os cuidados ao binômio mãe-bebê ${ }^{9,20}$. Pesquisadores apontam que profissionais de saúde, de forma geral, não abordam questões relacionadas ao uso de substâncias (lícitas ou ilícitas) durante o acompanhamento pré-natal. Tal fato pode ser decorrente da dificuldade na identificação do uso, visto que alguns de seus sintomas se confundem com patologias recorrentes da gestação; além da falta de tempo no atendimento prestado, registros inconsistentes e da inexperiência na abordagem sobre uso de substâncias psicoativas ${ }^{22}$. Logo, por meio da educação permanente, busca-se transformar práticas por meio de problematizações dos modos de cuidados vigentes, visando que profissionais de saúde possam desenvolver habilidades para realizar o rastreamento de usuárias. Assim, se favorece a intervenção precoce, criando a possibilidade de acesso a serviços especializados de tratamento e alternativas de enfrentamento ao uso de drogas de abuso na gestação, evitando ou amenizando complicações maternas e neonatais 6,9,23,24 .

A Organização Mundial da Saúde (OMS), visando auxiliar profissionais para melhor assistirem as mulheres grávidas que tenham algum distúrbio com substâncias psicoativas, desenvolveu diretrizes para a identificação, gestão e transtornos por uso de substâncias na gravidez. Tais diretrizes priorizam a prevenção, buscando reduzir e/ou interromper o uso dessas substâncias durante a gravidez e no período pós-parto, como forma de otimizar a saúde e o bem-estar do binômio mãe-bebê ${ }^{25}$.

No sentido preventivo, a educação em saúde destaca-se como uma importante estratégia de cuidado, uma vez que favorece o desenvolvimento da consciência crítica e reflexiva das gestantes e puérperas ao abrir espaço para o compartilhamento de um saber que contribui para o próprio autocuidado ${ }^{26}$. Quando desenvolvida no âmbito grupal, torna-se possível a criação de um espaço privilegiado a consolidação de uma rede de apoio e de troca de experiências, promovendo confiança entre gestantes/puérperas usuárias de substâncias psicoativas e profissionais, facilitando a adesão às condutas assistenciais ${ }^{27,28}$.

Em serviços de saúde cujos quais o tempo de atendimento e as condições de trabalho interferem no rastreamento e realização de uma avaliação completa, podem ser desenvolvidas intervenções mais breves. Tais intervenções podem abordar hábitos, recolhendo informações acerca do uso; aconselhar o cessamento e seus benefícios, assim como avaliar a motivação para realizá-lo; apoiar independente da escolha e acompanhar a evolução do processo identificando eventuais dificuldades que possam aparecer, inclusive a recaída no uso ${ }^{29}$.

Além disso, para dinamizar o rastreio do uso de substâncias, os profissionais podem utilizar-se de instrumentos padronizados com o Alcohol, Smoking and Substance Involvement Screening Test (ASSIST), o Alcohol Use Disorder Identification Test (AUDIT) e o Drug Abuse Screening Test (DAST) ${ }^{20}$. Esses instrumentos possuem grande valor, especialmente no período gravídico-puerperal em que a mulher está mais sensibilizada em relação aos efeitos nocivos que tais substâncias podem acarretar ao seu filho. Além disso, são de fácil compreensão, rápida aplicação, explora os diferentes tipos de substâncias e possibilita que os profissionais deem uma devolutiva a usuária, tornando-se uma estratégia de captação precoce e possível acompanhamento longitudinal ${ }^{30}$.

Por meio da captação precoce na atenção pré-natal, com acolhimento sensível às demandas e especificidades das usuárias, é possível ofertar um cuidado integral à mulher a partir de uma assistência multiprofissional. Pesquisadores apontam que mulheres grávidas dependentes de opioides podem ter ingestão inadequada de micronutrientes durante a gravidez, apontando como forma de cuidado o tratamento medicamentoso assistido. Este tratamento visa um melhor acompanhamento nutricional, atentando para um crescimento e desenvolvimento saudável para o feto, além de um aporte adequado para a mulher ${ }^{21}$. 
Outra estratégia de cuidado identificada nos artigos foi a Terapia de Substituição, que teve início com a técnica de prescrição controlada de heroína e morfina. Entretanto, com os avanços farmacológicos, novas substâncias começaram a fazer parte do rol de drogas de substituição, como é o caso da metadona e do composto buprenorfina-naloxona, utilizadas no tratamento para a dependência de opiáceos, tornando-se uma forma de redução de danos ${ }^{31}$. Uma pesquisa realizada na Escócia teve como objetivo analisar o impacto da Terapia de Substituição de opioides em usuárias de drogas que estavam esperando ou que tiveram recentemente um filho. Essa pesquisa apontou tal terapia como uma forma de proteção para a prole, passível às questões sociais e com implicações sobre a custódia do filho. Analogamente, implica também na manutenção da abstinência, minimizando o estigma da usuária de substância ${ }^{18}$.

Já a Terapia Cognitiva Comportamental, uma abordagem terapêutica estruturada, é focada no momento presente e utilizada para tratar problemas emocionais diversos, podendo ser empregada como uma forma alternativa de cuidado às mulheres que utilizam substâncias psicoativas no período gravídico-puerperal. Este tipo de abordagem é de custo moderado, além contribuir para a promoção de abstinência, permitindo uma compreensão mais abrangente da intervenção às mulheres que abusam de substâncias ${ }^{19,32}$.

Neste sentido, evidencia-se que o cuidado à mulher usuária de substâncias psicoativas no período gravídicopuerperal exige preparo dos profissionais de saúde; tanto para a captação precoce ao pré-natal quanto para a detecção e a intervenção adequadas que incluam ações contextualizadas e efetivas às gestantes e puérperas.

\section{Limitações do estudo}

Como limitação, aponta-se o fato de a presente revisão ter analisado a produção de conhecimento em um número restrito de bases, necessitando, assim, que o tema seja explorado por meio da busca nas demais bases de dados disponíveis.

\section{CONCLUSÃO}

A presente revisão apontou que há uma lacuna na literatura em relação às estratégias de cuidado às gestantes e puérperas usuárias de substâncias psicoativas. Os artigos analisados reconhecem fragilidades na atuação dos profissionais de saúde na abordagem e assistência às gestantes e puérperas usuárias, o que aponta para a necessidade de implementar atividades de educação permanente para qualificar a captação precoce e ofertar acolhimento sensível às demandas e especificidades de saúde dessas mulheres.

A bibliografia ressalta ainda a necessidade de investimento em estratégias estruturadas, tais como a Terapia Cognitivo Comportamental, visto que aborda problemas emocionais de largo espectro além de ser uma forma mais econômica de tratamento; bem como no uso da Terapia Substitutiva, que promove autonomia referente ao uso de substâncias psicoativas. É preciso atentar também para o acompanhamento nutricional, visto que mulheres grávidas usuárias de substâncias psicoativas tendem a realizar a ingestão inadequada de micronutrientes durante a gravidez, culminando em atrasos no desenvolvimento, no crescimento fetal e em disfunções metabólicas maternas.

As estratégias elencadas na presente revisão apontam possibilidades à prática dos profissionais que assistem as usuárias de substâncias psicoativas no período gravídico-puerperal. Isso ocorre por se tratar de um cuidado complexo, que envolve tanto o acompanhamento pré-natal, visando os desfechos maternos e fetais, quanto o cuidado à mulher usuária de substâncias psicoativas e suas especificidades.

\section{REFERÊNCIAS}

1. Teixeira MB, Lacerda A, Riberio JM. Potentialities and challenges of an intersectoral public policy on drugs: "With Open Arms" Program of São Paulo, Brazil. Physis [Internet] 2018 [cited 2020 Nov 25]; 28(3). DOI: http://dx.doi.org/10.1590/S010373312018280306

2. ONU. Organização das Nações Unidas. Agenda 2030. [Internet] 2015 [cited 2020 Jan 23]. Available from: https://nacoesunidas.org/pos2015/agenda2030/.

3. Lopes AB, Vieira ALN, Ribeiro CC, Andrade DAR, Generoso LN, Diamantino FC, et al. Drug use in pregnancy. Rev. Med. Minas Gerais. [Internet] 2011 [cited 2020 Jan 26]; 21(4): (2 Supl 4): S1-S11. Available from: http://rmmg.org/artigo/detalhes/913

4. Das $P$, Horton R. The global drug problem: change but not progression. The Lancet [Internet] 2019 [cited 2020 Jan 26]; 394(10208):1488-90. DOI: https://doi.org/10.1016/S0140-6736(19)32275-5.

5. Rocha PC, Alves MTSSB, Chagas MDC, Silva AAM, Batista RFL, Silva RA. Prevalence of illicit drug use and associated factors during pregnancy in the BRISA cohort. Cad. Saúde Pública [Internet] 2016 [cited 2020 Jan 26]; 32(1). DOI: http://dx.doi.org/10.1590/0102-311X00192714.

6. Mais JA, Rodrigues AL, Souza DR, Figueiredo MB. U Drug use by women during the gestational period. Revista Enfermagem Contemporânea, [Internet] 2019 [cited 2020 Jan 26]; 8(1): 25-32. DOI: http://dx.doi.org/10.17267/2317-3378rec.v8i1.1744

7. Pereira CM, Pacagnella RC, Parpinelli MA, Andreucci CB, Zanardi DM, Souza R, Angelini CR, Silveira C, Cecatti JG. Drug Use during Pregnancy and its Consequences: A Nested Case Control Study on Severe Maternal Morbidity. Rev. Bras. Ginecol. Obstet. [Internet] 2018 [ cited 2020 Nov 24]; 40(9): 518-26. Avaliable from: https://www.scielo.br/pdf/rbgo/v40n9/0100-7203-rbgo-4009-00518.pdf. 
8. Lemos IRO, Taveira GMT. Prenatal conditions of pregnant women users legal drugs that calved in reference hospital in Singapore, 2013-2014. Revist. Port.: Saúde e Sociedade [Internet] 2016 [cited 2020 Jan 26]; 1(2):130-45. Available from: https://www.seer.ufal.br/index.php/nuspfamed/article/download/2366/1951.

9. Kassada DS, Marcon SS, Waidman MAP. Perceptions and practices of pregnant women attended in primary care using illicit drugs. Esc. Anna Nery [Internet] 2014 [cited 2020 Jan 26]; 18(3): 428-34. Avaliable from: http://www.scielo.br/pdf/ean/v18n3/en_1414-8145-ean-18-03-0428.pdf.

10. Ministério da Saúde (Brasil). Secretaria de Atenção à Saúde. Departamento de Atenção Básica. Atenção ao pré-natal de baixo risco. Brasília: Editora do Ministério da Saúde, 2012. Avaliable from: http://bvsms.saude.gov.br/bvs/publicacoes/cadernos_atencao_basica_32_prenatal.pdf.

11. Febrasgo. Drogas ílicitas e gravidez. Femina [Internet] 2018 [cited 2020 Jan 26]; 46(1): 10-18. Avaliable from: https://www.febrasgo.org.br/media/k2/attachments/VolZ46Z-Zn1-Z2018.pdf.

12. Santos HTS, Oliveira GS, Soares PCF, Araújo WA, Almeida EUA, Oliveira MMLR. Harms in tobacco use in pregnancy and its complications to the fetus los daños del uso de tabaco en el embarazo y sus complicaciones al feto. Rev. enferm. UFPE on line [Internet] 2015 [cited 2020 Nov 26]; 9(Supl. 9):9978-82. DOI: https://periodicos.ufpe.br/revistas/revistaenfermagem/article/download/10796/11958

13. Reitan T. Substance abuse during pregnancy: a 5-year followup of mothers and children. Drugs: Education, Prevention and Policy [Internet] 2019 [cited 2020 Nov 24]; 26(3): 219-28. Avaliable from: https://www.tandfonline.com/doi/pdf/10.1080/09687637.2018.1432568?needAccess=true.

14. Camargo PO, Martins MFD. The effects of crack in pregnancy and babies of addicted mothers: A literature review. Cad. Ter. Ocup. [Internet] 2014 [cited 2020 Jan 26]: 22(1): 161-69. DOI: http://dx.doi.org/10.4322/cto.2014.042.

15. Ministério da Saúde (Brasil). Secretaria de Ciência, Tecnologia e Insumos Estratégicos. Departamento de Ciência e Tecnologia. Agenda nacional de prioridades de pesquisa em saúde. Brasília: Editora do Ministério da Saúde, 2015 [cited 2020 Jan 26]. Avaliable from: http://bvsms.saude.gov.br/bvs/publicacoes/agenda_prioridades_pesquisa_ms.pdf.

16. Sousa LMM, Vieira CMAM, Severino SSP, Antunes AV. A metodologia de revisão integrativa da literatura em enfermagem. Revista investigação em Enfermagem [Internet] 2017 [cited 2020 Nov 25]:17-26. DOI: https://doi.org/10.1590/S010407072008000400018

17. Ministério da Saúde (Brasil). Portaria no 1.459, de 24 de junho de 2011. Institui no âmbito o Sistema Único de Saúde -SUS- a rede Cegonha. Brasil: Ministério da Saúde, 2011.

18. Chandler A, Whittaker A, Burley SC, Willians N, Mcgorm K, Mathews G. Substance, structure and stigma: Parents in the UK accounting for opioid substitution therapy during the antenatal and postnatal periods. Int. j. drug policy [Internet] 2013 [cited 2020 Jan 26]; 24(6): 35-42. DOI: http://dx.doi.org/10.1016/j.drugpo.2013.04.004.

19. Xu X, Yonkers K, Ruger J. Costs of a Motivational Enhancement Therapy Coupled with Cognitive Behavioral Therapy versus Brief Advice for Pregnant Substance Users. PLoS One [Internet] 2014 [cited 2020 Jan 26]; 9(4): e95264. Avaliable from: https://www.ncbi.nlm.nih.gov/pmc/articles/PMC3997437/pdf/pone.0095264.pdf.

20. Yotebieng Kelly, Agot K, Rota G, Cohen C, Syvertsen J. A Qualitative Study of Substance use during Pregnancy: Implications for Reproductive Healthcare in Western Kenya. Afr. J. Reprod. Health [Internet] 2016 [cited 2020 Jan 26]; 20(4): 51-59. Avaliable from: https://www.ncbi.nlm.nih.gov/pmc/articles/PMC6076375/pdf/nihms-982903.pdf.

21. Shrestha S, Jimenes E, Garrison L, Pribis P, Raisch D, Stephen J, et al. Dietary Intake Among Opioid Dependent and Alcohol Using Pregnant Women. Subst. Use Misuse [Internet] 2018 [cited 2020 Jan 26]; 53(2): 260-69. Avaliable from: https://www.ncbi.nlm.nih.gov/pmc/articles/PMC5607070/pdf/nihms871814.pdf.

22. Varela DSS, Sales IMM, Silva FMD, Monteiro CFS. Health network assisting users of alcohol, crack, and other drugs red de salud en la atención a los usuarios de alcohol, crack y otras drogas. Esc. Anna Nery [Internet] 2016 [cited 2020 Mar 24]; 20(2):296302. Available from: https://www.scielo.br/scielo.php?pid=S1414-81452016000200296\&script=sci_arttext\&tlng=en.

23. Batista CB, Vasconcelos MPN, Vecchia MD, Queiroz IS. Permanent education on harm reduction: the experience of Psychosocial Care Course in Alcohol and other Drugs. Interface [Internet] 2019 [cited 2020 Nov 25]; 23: e180071. DOI: http://dx.doi.org/10.1590/interface.180071.

24. Kassada DS, Marcon SS, Pagliarini MA, Rossi RM. Prevalence of drug abuse among pregnant women. Acta Paul. Enferm. [Internet] 2013 [cited 2020 Jan 26]; 26(5):467-71. Avaliable from: http://www.scielo.br/pdf/ape/v26n5/en_a10v26n5.pdf.

25. Organização Mundial de Saúde. Guidelines for the identification and management of substance use and substance use disorders in pregnancy. WHO; 2014 [cited 2020 Nov 25]. Avaliable from: https://www.who.int/publications/i/item/9789241548731.

26. Paiva MVS, Soares AMM, Lopes ARS, Santos KCB, Sardinha AHL, Rolim ILTP. Health education with pregnant and puerperal women: an experience report. Revista Recien [Internet] 2020 [cited 2020 Jan 24]; 10(29):112-119. DOI: https://doi.org/10.24276/rrecien2358-3088.2020.10.29.112-119.

27. Regra GL, Salerno GRF, Fernandes SMS. Healthy education for pregnant women and postpartum women. Revista Pesquisa em Fisioterapia [Internet] 2017 [cited 2020 Nov 24]; 7(3):351-8. Available from: https://www5.bahiana.edu.br/index.php/fisioterapia/article/view/1477/941.

28. Alves FLC, Castro EM, Souza FKR, Lira MCPS, Rodrigues FLS, Pereira LPP. Group of high-risk pregnant women as a health education strategy. Rev. Gaúcha Enferm. [Internet] 2019 [cited 2020 Nov 24]; 40(1):e20180023. DOI: https://doi.org/10.1590/1983-1447.2019.20180023.

29. Nunes $E$, Narigão M. Cessão tabágica na gravidez - Guia para os profissionais de saúde. Programa Nacional para a Prevenção e Controlo do Tabagismo 2015. 2015 [cited 2020 Nov 24]. Available from: https://www.dgs.pt/programa-nacional-para-a- 
prevencao-e-controlo-do-tabagismo/relatorios-e-publicacoes/cessacao-tabagica-na-gravidez-guia-para-profissionais-desaude1.aspx.

30. Fornigoni MLOS, Corneiro APL, Avallone DM. Intervenção breve: princípios básicos e aplicação passo a passo. Senad [Internet] 2017 [cited 2020 Jan 26]. Available from: http://www.aberta.senad.gov.br/medias/original/201704/20170424-095204-001.pdf.

31. Gomes TB, Vecchia MDV. Harm reduction strategies regarding the misuse of alcohol and other drugs: a review of the literature. Ciênc. saúde colet [Internet] 2018 [cited 2020 Jan 26]; 23(7):2327-38. Avaliable from: http://www.scielo.br/pdf/csc/v23n7/en_1413-8123-csc-23-07-2327.pdf.

32. Oppermann CMO, Lewgoy LB, Araujo RB. Dialectical behavior therapy for drugs addiction tatiens. Rev. bras.ter. cogn [Internet] 2015 [cited 2020 Jan 26]; 11(2): 113-8. DOI: http://dx.doi.org/10.5935/1808-5687.20150016. 